\title{
Plastic Electronics based Conformable Electronic Circuits
}

\author{
F. Bossuyt, S. Dunphy, J. Vanfleteren, J. De Baets, K. Pacheco Morillo, J. Van den Brand
}

CMST - IMEC/Ghent University

Technologiepark 914 A, 9052 Zwijnaarde, Belgium

Frederick.bossuyt@elis.ugent.be,+32(0)92645354

\begin{abstract}
In this contribution, results on technology developments are presented aiming to realize conformable electronic systems based on plastic electronics technologies. Focus of the developments is on low cost with an acceptable reliability in function of the end-application. The feasibility of this technology is demonstrated by digging into the different process steps and their characteristics. A number of demonstrators (large-area conformable illumination tiles) have been realized and are discussed. The major part of this contribution is on the mechanical characterization of these plastic electronics based technologies.
\end{abstract}

\section{Introduction}

In the last decade, a high number of technologies to realize conformable electronic systems have been developed by a number of research institutes [1-8]. A wide variety of different processes exists nowadays to realize such systems, where each technology has its own particular characteristics and targeted application fields. Each technology has its advantages and disadvantages in terms of processability, reliability, scalability and cost.

In this contribution, we discuss a technology with the aim to realize roll-to-roll, large area conformable systems mainly for lighting applications situated in the consumer health market and design industry. Substrate materials like PET/PEN in combination with printed (e.g. silver paste) or etched (e.g. from laminated copper foil) meandering metal structures are used as starting point. The foils obtain their conformability by structuring them using laser technology.

\section{Fabrication process}

In [5-8], the Stretchable Molded Interconnect technology has been proposed to realize a stretchable circuit (SMI-technology). Compared to traditional printed circuit boards (PCBs) or flexible circuit boards (FCBs), the SMI technology pushes the conformability of electronic circuitry to a higher level, by allowing it to stretch in different directions. To obtain this stretchability, the circuit is subdivided in several non-stretchable functional islands (including the non-stretchable, conventional electronic components), which are interconnected with stretchable, meandered in-plane interconnections. The stretchable circuit is finally encapsulated in an elastomer, resulting in a stretchable module that can e.g. be integrated in textile [9].

The technology presented here is based on the SMItechnology, but uses some other materials and processes. The process is depicted in Figure 1. We start from a laminate including $18 \mu \mathrm{m}$ copper laminated onto a plastic foil (PET/PEN). The copper is lithographically patterned and etched into stretchable interconnect patterns and structures for the component islands. In this way, the metallic conductors are defined onto the plastic foil. Another approach is to screenprint silverpaste onto a bare foil (PET/PEN), to define similar structures for the stretchable circuit. The confomability of the foil is obtained by laserstructuring the foil. Therefore, the foil is first placed on a temporary carrier [5] to hold the structures in place during structuring and secondly, the residual foil parts that do not end up in the final device are peeled out.

Electronic functionality is achieved by assembling electronic components (e.g. LEDs, microcontrollers, passives) by means of conductive pastes (ICAs/ACAs).

An encapsulation in a stretchable polymer by liquid injection moulding PDMS or laminating PU foils completes the process (Figure 1).

In Figure 2, a 3 by 3 stretchable matrix of light tiles is shown. A light tile here is a $2 \mathrm{~cm}$ by $2 \mathrm{~cm}$ foil island, with 2 top emitting white LEDs in the middle of the island. The matrix is laminated into a polyurethane, Krystalflex 399 (Huntsman). In the picture, it is shown that this device is conformable.
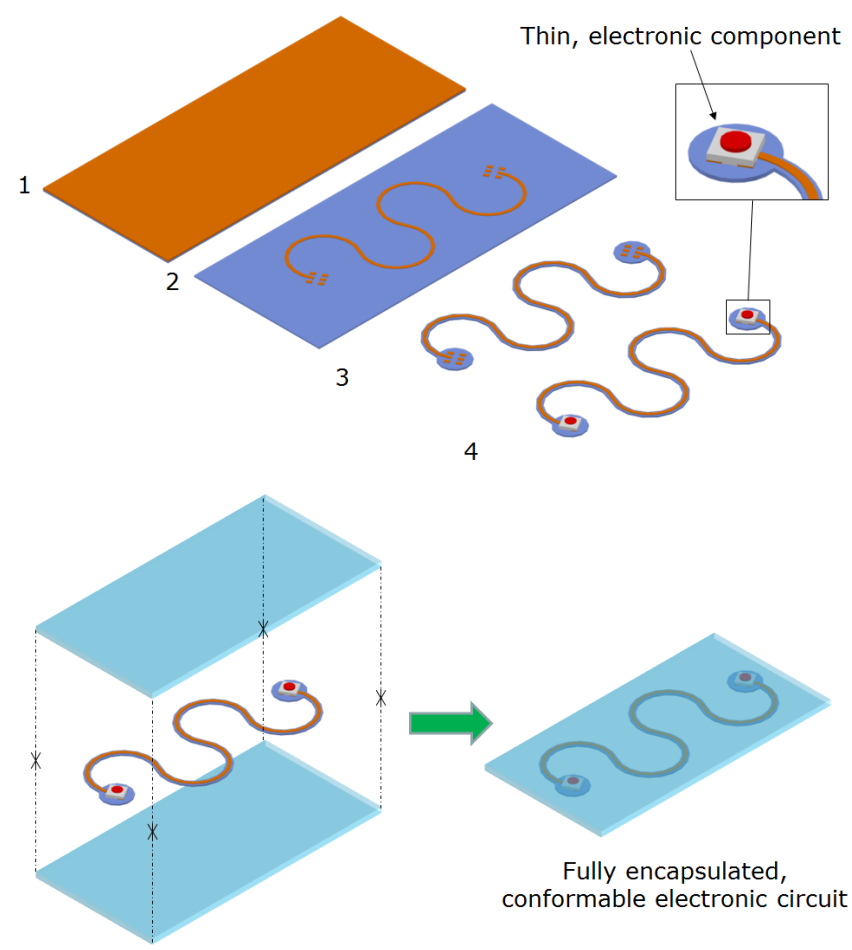

Figure 1 Process flow for realizing plastic electronic based conformable circuits 


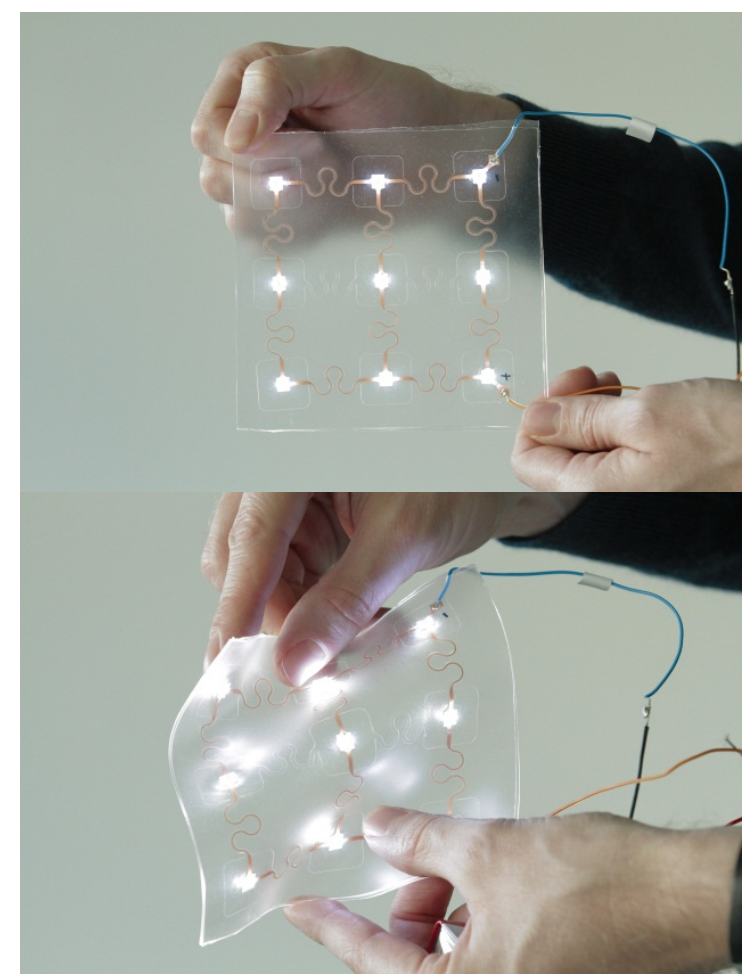

Figure 2 Realized demonstrator and its conformability

\section{Mechanical performance}

Previously, the mechanical performance during cyclic stretching of the SMI technology has been analyzed. This for bare copper meanders [6] and for polyimide supported copper tracks [7]. In previous publications, we demonstrated that mechanical support for the meanders improves their lifetime significantly. The polyimide layer effectively redistributes the plastic strain in the metal and therefore improves the reliability of the stretchable interconnect.

Again, we want to characterize the performance of these new types of stretchable interconnects, now with PET/PEN support. Therefore, an extensive study on the lifetime of stretchable interconnects in relation with design and technology parameters, has been executed. Testvehicles as shown in Figure 3 have been made with variation in meander track width (wide tracks: $\sim 230 \mu \mathrm{m}$, narrow tracks: $100 \mu \mathrm{m}$ ). The meander is a horseshoe as defined in [6] with a meander angle of $30^{\circ}$. In the testvehicles, the copper meander is supported by a laserstructured foil where the supporting foil type is PET, PEN or PI. The width of the supporting meander is typically $450 \mu \mathrm{m}$ where the metallic interconnect is in the middle. As starting point for the production, commercially available laminates were used (foils with $50 \mu \mathrm{m}$ PET support: GTS Laminates 556920ED0610, foils with $125 \mu \mathrm{m}$ PET support: GTS Laminates 556950ED-0610, foils with $50 \mu \mathrm{m}$ PEN support: GTS Laminates 566920ED-0610, foils with $125 \mu \mathrm{m}$ PEN - GTS Laminates 566950ED-0610, foils with $50 \mu \mathrm{m}$ PI support: Upisel-N SE 1220). It is important to note that the PET/PEN based laminates have an adhesive between foil and copper (PU based adhesive). For the PI, the laminate is adhesiveless.

PDMS (Sylgard 186, Dow Corning) was used as encapsulant, where a dedicated mould was fabricated for encapsulation in order to obtain the thicker clamping structures at the edge (cross-section of sample has a dog bone shape). PDMS thickness in the meander zones is $\sim 1$ $\mathrm{mm}$.

The average initial resistance of the tracks is $\sim 1,26 \Omega$ for PET supported, $230 \mu \mathrm{m}$ wide copper tracks, $\sim 1,06 \Omega$ for PEN supported $230 \mu \mathrm{m}$ wide copper tracks, $\sim 2,56 \Omega$ for PEN supported $100 \mu \mathrm{m}$ wide copper tracks. The cyclic stretch tests were performed at $10 \%, 15 \%$ and $20 \%$ elongation with a strainrate of $10 \% / \mathrm{sec}$. For the polyimide based samples, the elongations were taken higher because the time-to-failure of these samples is much longer for small elongations $(20 \%, 40 \%, 60 \%)$.

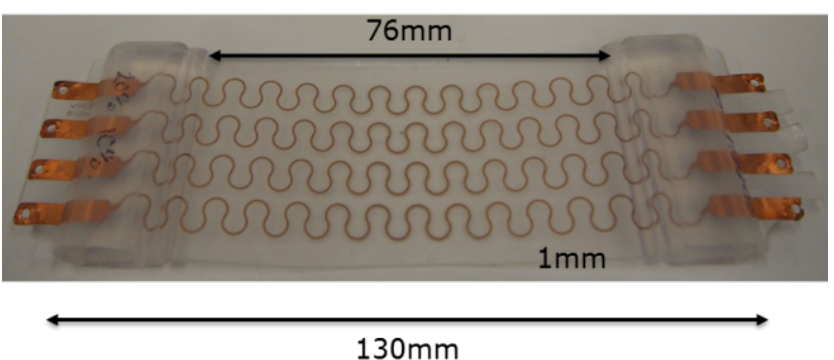

\section{Figure 3 Encapsulated meander tracks for cyclic endurance testing}

During cycling stretching, for all samples, the resistance remains constant. From a certain point, the resistance gradually increases due to micro-cracks (Figure 5) in the copper due to plastic deformation. From microscope analyses, it is observed that the support material doesn't break for the examined elongations. The resistance behavior during cyclic stretching is depicted in Figure 4.

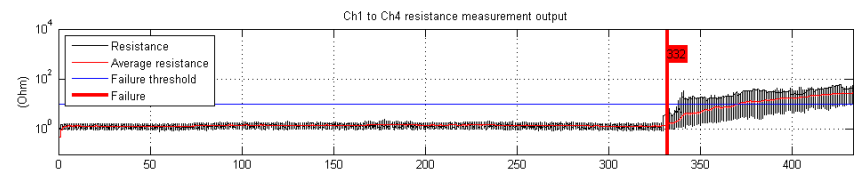

Figure 4 Resistance behavior of testsample (50 $\mu \mathrm{m}$ PET support)

In Figure 5, the typical crack formation at the top and bottom of the meanders is shown, spread over a wider distance compared to unsupported copper tracks. This is in line with the places of highest strain occurring during stretching as reported earlier [7].

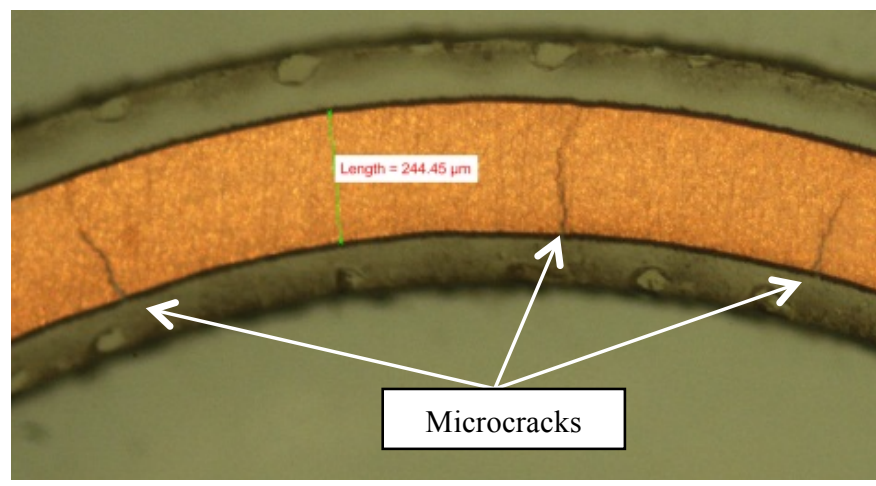

Figure 5 Crack formation in $18 \mu \mathrm{m}$ ED copper with $50 \mu \mathrm{m}$ PET support 


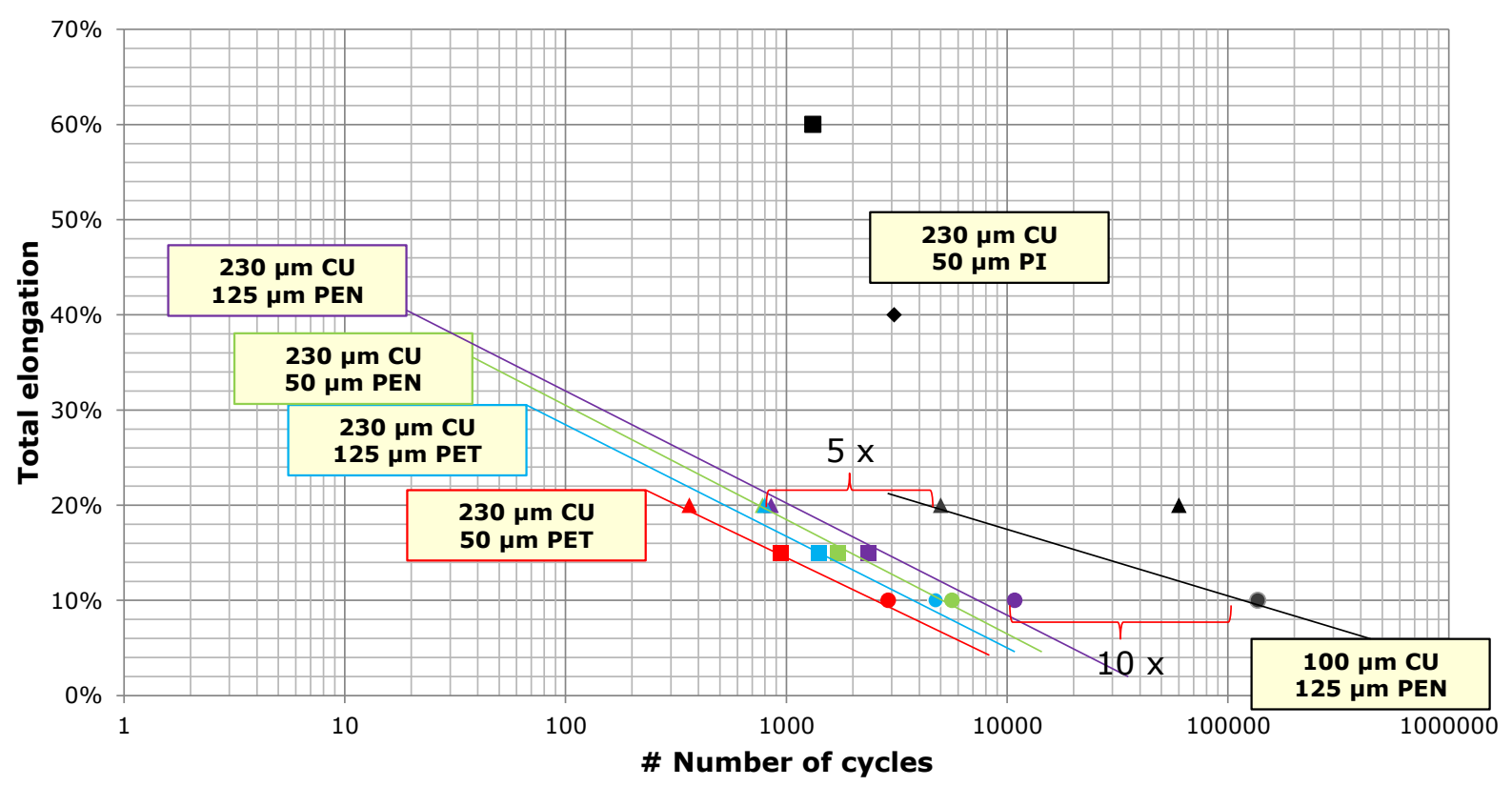

Figure 6 Number of stretch cycles before electrical failure in function of total elongation and materials/design

Another failure that is observed is the interfacial delamination between the meander and the encapsulant (Figure 7). This phenomenon has already been described in [7].

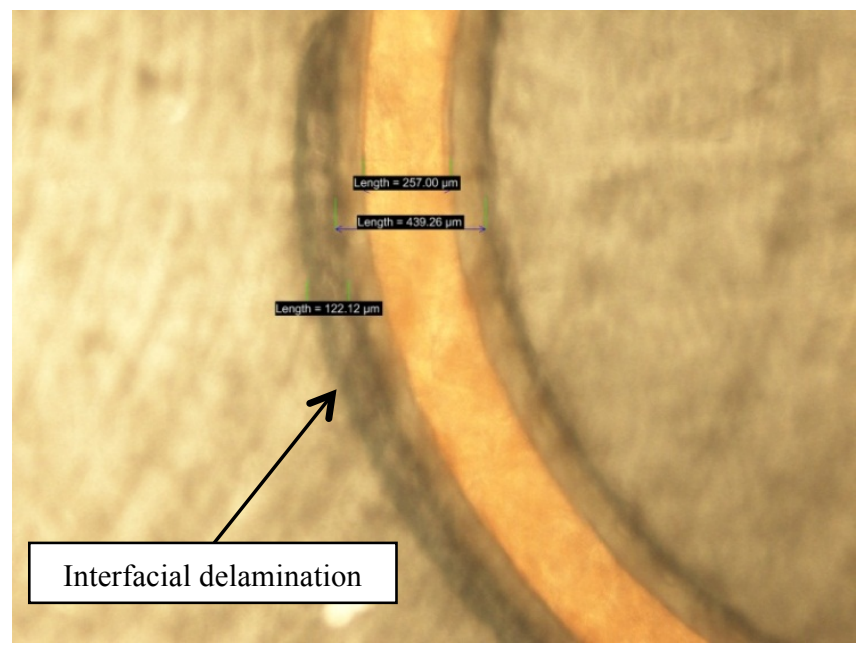

Figure 7 Interfacial delamination occurring in all samples at sides of meanders

Per sample, there are 4 tracks. The average number of cycles to failure is plotted in Figure 6. We defined the number of cycles to failure by the onset point for electrical failure, which we have fixed at an increase of track resistance above $10 \Omega$. If one looks to Figure 6 , it's clear that the narrower copper tracks perform better compared to the wider tracks. They reach 5 to 10 times more cycles before electrical failure (for $125 \mu \mathrm{m}$ PEN). If one compares the performance of the different foil types, it's clear that the polyimide supported tracks perform the best. Then PEN performs better compared to PET. For the PET/PEN supported stretchable interconnects, the thickness of the support material was compared (50 $\mu \mathrm{m}$ and $125 \mu \mathrm{m})$. The effect is less pronounced but it seems that the thicker the support material, the better the performance. If one looks to the plastic based interconnects, one can see that for elongations up to $10 \%$, depending on the design and technology, the interconnections can survive up to 100.000 cycles, without diminishing in electrical conductivity. For the polyimide based samples, we stopped the $20 \%$ elongation test at 60.000 cycles and analyzed the sample. No starting failures were observed so far. For higher elongations, we obtained 3078 cycles for $40 \%$ elongation and 1318 cycles for $60 \%$ elongation. In both cases, the PDMS encapsulation failed (tearing).

\section{Mechanical performance analysis}

The clarification of the obtained results is still an ongoing study, not all aspects are yet fully understood. The following factors affect the lifetime:

Young's modulus of support material (PET, PEN, PI): From simulations, we have learned that the higher the Young's modulus for the supporting material, the lower the plastic strain is in the copper [7]. This confirms with the experiments, where the lifetime of the PET $<$ PEN $<$ PI. This is in accordance with the Young's modulus of PET < PEN < PI. But, the difference in Young's modulus between PEN and PI is not high enough to clarify this huge difference in lifetime.

Thickness of support material: From simulations, we know the thicker the support material, the lower the plastic strain in the copper [7]. This is in accordance with the experimental results, but the impact of increasing the thickness is low.

Support material width (compared to copper track width): The stress on the meander during stretching is originating from the tensile stress of the PDMS on the track and the tensile stress occurring due to the opening of the meander. Simulations have shown that the wider the support material 
compared to the copper width, the lower the plastic strain in the copper [7]. This is confirmed by the experiments where we observe a clear difference between lifetime of $230 \mu \mathrm{m}$ and $100 \mu \mathrm{m}$ tracks.

Adhesive/Adhesiveless laminate: From experiments, we observe that adhesiveless laminates perform much better compared to the ones with adhesive. Due to the full encapsulation, it's hard to observe the status of the adhesive after cyclic stretching. More study is needed and will be performed to know the impact of the adhesive between copper and support. One can think of samples which are halfly encapsulated in order to study the copper/adhesive/PE(T)(N) interface during crack formation. The stress on the tracks will be somehow different due to the half encapsulation, but it can give us an indication of the crack initiation and propagation.

Copper constitution: SEM pictures have been made of cross-sections of the different substrates used. We have observed that the laminated copper has a more vertical, slice structure compared to the electrodeposited copper on PI which has a more granular structure. To know the effect of the copper constitution, we could use the same halfly encapsulated samples to study the copper performance during crack propagation.

\section{Conclusions}

In this contribution, we have presented technology developments to realize conformable electronic systems based on plastic electronics technologies. The technology to make conformable circuits is based on the earlier reported SMI technology, but now cheaper materials and production methods are used to fabricate conformable circuits. Mechanical characterization of these plastic electronics' based technologies has been performed. This by executing cyclic endurance tests on dedicated test vehicles including parallel meander tracks. Different materials and designs have been evaluated in order to be able to make design decisions in function of end applications. From the mechanical performance analysis and in line with previous data from publications, we note that the higher the Young's modulus of the meander support material, the higher the lifetime of the interconnects $(\mathrm{PET}<\mathrm{PEN}<\mathrm{PI})$. A thicker supporting material leads to a (not so pronounced) higher lifetime. The support material width has a big impact on the lifetime. Furthermore, from the experimental results we expect that the adhesiveless nature and the copper constitution of the used foils, play a major role in the lifetime. But, these effects need more study to fully understand their impact on the lifetime.

\section{References}

1. Mandlik, P., Lacour, SP., Li, WJ., Chou, SY., Wagner, S., Fully elastic interconnects on nanopatterned elastomeric substrates. IEEE Electron Dev Lett 2006;27:650-2.Kim, DH., Rogers, JA. Stretchable electronics: materials strategies and devices. Adv Mater 2008;20:4887-92.

2. Sekitani, T., Someya, T., Stretchable, large-area organic electronics. Adv Mater 2010;22:1-19.

3. Kim, D., Xiao, J., Song, J., Huang, Y., Rogers, JA. Stretchable, curvilinear electronics based on inorganic materials. Adv Mater 2010;22:1-17.

4. Lacour, SP., Jones, J., Suo, Z., Wagner, S. Design and performance of thin metal film interconnects for skinlike electronic circuits. IEEE Electron Dev Lett 2004;25:179-81.

5. F. Bossuyt, T. Vervust, F. Axisa, J. Vanfleteren, A new low cost, elastic and conformable electronics technology for soft and stretchable electronic devices by use of a stretchable substrate. In: Microelectronics and Packaging Conference EMPC 2009, pp. 697-702.

6. F. Bossuyt, J. Guenther, T. Löher, M. Seckel, T. Sterken, J. de Vries, Cyclic endurance reliability of stretchable electronic substrates, Microelectronics Reliability, Volume 51, Issue 3, March 2011, pp. 628635

7. Hsu Yung-Yu, Gonzalez M., Bossuyt F., Vanfleteren J., De Wolf I., Polyimide-Enhanced Stretchable Interconnects: Design, Fabrication, and Characterization, IEEE Transactions On Electron Devices, Vol. 58, No. 8, AUGUST 2011

8. F. Bossuyt, T. Vervust, F. Axisa, and J. Vanfleteren, Improved stretchable electronics technology for large area applications, MRS Proceedings, vol. 1271. San Francisco, CA, USA: Materials Research Society (MRS), April 2010, pp. 1-7

9. T. Vervust, F. Bossuyt, F. Axisa, and J. Vanfleteren, Stretchable and washable electronics for embedding in textiles, MRS Proceedings, vol. 1271, San Francisco, CA, USA: Materials Research Society (MRS), April 2010, pp. 1-6 